\title{
Review
}

\section{Transthoracic Ultrasonography for the Respiratory Physician}

\author{
Coenraad F.N. Koegelenberg Florian von Groote-Bidlingmaier Chris T. Bolliger \\ Division of Pulmonology, Department of Medicine, Stellenbosch University and Tygerberg Academic Hospital, \\ Cape Town, South Africa
}

\author{
Key Words \\ Lung cancer $\cdot$ Pleural disease $\cdot$ Transthoracic biopsy $\cdot$ \\ Ultrasound
}

\begin{abstract}
Transthoracic ultrasonography is still not utilized to its full potential by respiratory physicians, despite being a well-established and validated imaging modality. It allows for an immediate and mobile assessment that can potentially augment the physical examination of the chest. Ultrasound (US)assisted procedures can be performed by a single clinician with no sedation and with minimal monitoring, even outside of theatre. The main indications for the use of transthoracic US are: the qualitative and quantitative description of pleural effusions, pleural thickening, diaphragmatic dysfunction and chest-wall and pleural tumours. It may also be used to visualise lung tumours and other parenchymal pulmonary processes provided they abut the pleura. It is at least as sensitive as chest radiographs as far as the detection of a pneumothorax is concerned. It is the ideal tool to assist with thoracocentesis and drainage of effusions. The US-assisted fine-needle aspiration and/or cutting-needle biopsy of extrathoracic lymph nodes, lesions arising from the chest wall, pleura, peripheral lung and mediastinum, are safe and have
\end{abstract}

a high yield in the hands of chest physicians. US may also guide the aspiration and biopsy of diffuse pulmonary infiltrates, consolidations and lung abscesses, provided the chest wall is abutted. Advanced applications of transthoracic US include the diagnosis of pulmonary embolism.

Copyright $\odot 2012$ S. Karger AG, Basel

\section{Introduction}

Diagnostic ultrasonography is by no means a recent addition to the armamentarium of the clinician. Sonography of the abdomen was first performed in the 1940s. However, it took approximately half a century for respiratory physicians to appreciate the impact transthoracic ultrasound (US) could have on patient safety and diagnostic yield, and for the modality to be widely utilized [1-7]. As an imaging modality, transthoracic US has many advantages, the most significant being its immediate application at the point of care and its ability to augment the clinical assessment of the respiratory system $[4,8]$. It is relatively cheap, mobile, utilizes no radiation and has a short examination time [1-7]. Moreover, US-assisted procedures can be performed by a single clinician with no sedation and minimal monitoring, even potentially out-

\section{KARGER}

Fax +4161306 1234

E-Mail karger@karger.ch

www.karger.com
(C) 2012 S. Karger AG, Basel

$0025-7931 / 12 / 0844-0337 \$ 38.00 / 0$

Accessible online at:

www.karger.com/res
Coenraad F.N. Koegelenberg, MB ChB, FCP (SA), MRCP (UK), PhD

Division of Pulmonology, Department of Medicine

Stellenbosch University and Tygerberg Academic Hospital

PO Box 19063, Tygerberg, Cape Town 7505 (South Africa)

Tel. +27 21938 9243, E-Mail coeniefn@sun.ac.za 
side of theatre $[1-2,5]$. This review will give an overview of general technical aspects of transthoracic US, cover diagnostic procedures as well as US-assisted interventions and will conclude with a summary of current suggestions on basic competency in ultrasonography for the pulmonary specialist.

\section{Technical Aspects}

\section{Basic Physics and Principles}

'Ultrasounds' are sounds with a frequency above human hearing $[1,2]$. Transthoracic US scanning is generally performed in the frequency range of 2-10 megahertz $(\mathrm{MHz})$. The sound waves are produced and focussed by a piezoelectric transducer in a hand-held probe and transmitted into the thorax. US waves are propagated in liquid media (e.g. pleural effusions) or through tissues with a high water content (e.g. muscle, liver, consolidated lung or tumours). The majority of the acoustic waves are reflected when sounds encounter gas (e.g. aerated lungs or a pneumothorax) or solids (e.g. ribs), because of the significant difference in density $[1,2]$. This phenomenon is also known as acoustical impedance and is the reason why structures deeper than the visceral pleura are not discernable by means of ultrasonography in the non-diseased state $[1,2]$.

An echo is the reflected part of the sound waves, which is captured by the transducer and converted into electrical pulses, which are ultimately processed and transformed into a digital image. The intensity and distribution of the pixels appearing on the screen is determined by three characteristics of the echo, namely (1) its direction, (2) its intensity and (3) the time elapsed from emission to capture $[1,2]$. An echo will have greater intensity with an increasing difference in acoustic impedance between interfaces from which it is reflected. Fluid will appear black and solid organs will have variable echogenicity, depending on the fluid content and fraction of reflected waves, whereas air gives rise to random snow storm. The echogenicity of any lesion is defined relative to that of the liver, which is arbitrarily considered to be isoechoic [9].

\section{Ultrasound Equipment}

Most modern US units offer 2-dimensional, motionmode (M-mode) and colour flow Doppler scanning. Adequate transthoracic ultrasonography can, however, be performed by means of the most basic, entry-level, 2dimensional black-and-white US equipment. A low-fre- quency probe $(2-5 \mathrm{MHz})$ with a curvilinear shape for covering a large area is suitable for the initial screening of superficial and deeper structures, whereas a high-frequency probe $(5-10 \mathrm{MHz})$ with a linear shape is used for the refined assessment of an abnormal chest wall or pleural area. Higher frequency gives better resolution closer to the probe, but at the cost of less penetration [1-3]. Doppler and M-mode are not essential for routine thoracic examination.

A clinician utilizing US should be familiar with the basic controls, including the 'depth', 'gain' and 'freeze' functions [1-3]. The depth function is a digital zoom that defines what portion of the scanned image is displayed on the monitor at a certain magnification. The scale is displayed on a vertical axis. Obese subjects or patients with a large effusion may require a depth setting of up to $12 \mathrm{~cm}$. High-frequency scanning is performed at a maximum depth of around 3-4 cm. Ideally, the depth should be adjusted in such a way that the area of interest fills the digital screen. The gain is, in essence, a measure for the amplification of the echos and determines the brightness of the image, and should be adjusted to maximise the contrast between tissues. The freeze function allows for the capturing of still images, and to perform measurements with the appropriate keys and the trackball.

\section{Patient Positioning and Scanning}

The ideal patient position for scanning is a critical factor for obtaining optimal images $[1,3]$. Unlike the emergency medicine physician, who frequently utilizes transthoracic US as the initial imaging modality, the respiratory physician not infrequently has access to a patient's chest radiograph and computed tomography (CT) scan prior to performing a thoracic US examination. These investigations not only identify the area of interest, they also guide the positioning of the patient $[1,2]$. The posterior chest is best scanned with the patient in the sitting position using a bedside table as an armrest, whereas the lateral and anterior chest wall can be examined with the patient in either the lateral decubitus or even the supine position.

Emergency medicine physicians and intensive care specialists often hold the transducer in a longitudinal plane with the transducer indicator in a cephalad position [8]. Although this technique has a few potential advantages (e.g. greater sensitivity for pneumothoraces), superior visualisation of the pleura and peripheral lung tumours and consolidated lung can be achieved by examining along the intercostal spaces [1]. Pulmonary specialists therefore often utilize this technique. Raising the 


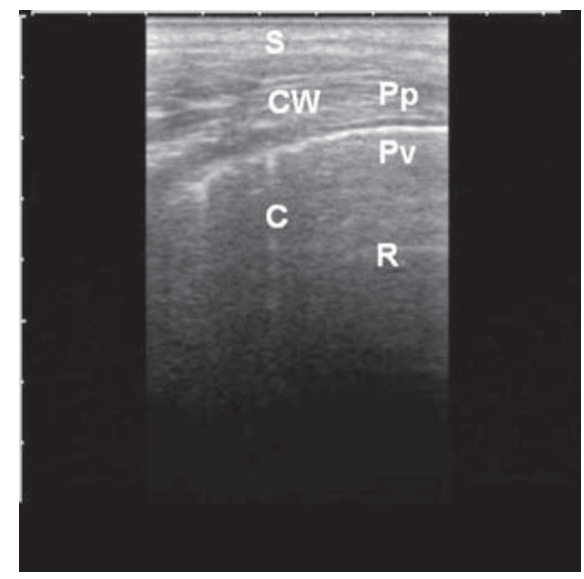

Fig. 1. The typical appearance of a normal chest on US (transverse image through the intercostal space with a high-frequency probe). The chest wall appears as multiple layers of echogenicity representing muscles and fascia. The visceral and parietal pleura are seen as echogenic bright lines that slide during respiration. Alines beneath the pleural lines imply an underlying air-filled lung. $\mathrm{C}=$ Comet-tail artefact; $\mathrm{CW}=$ chest wall; $\mathrm{Pp}=$ parietal pleura; $\mathrm{Pv}=$ visceral pleura; $\mathrm{R}=$ reverberation artefact; $\mathrm{S}=$ skin.

arm above the patient's head increases the intercostal space distance and aids in scanning in erect or recumbent positions. A patient can fold the arms across the chest in order to displace the scapulae when scanning the upper posterior thorax. Superior sulcus pathology can be visualised apically with the patient in the supine or sitting position.

Once the patient is adequately positioned and the area of interest is identified, liberal application of gel is the final step prior to scanning [1]. It is advisable to hold the probe like a pen with the outer part of the hand in contact with the skin, and to reduce ambient lighting. Experienced sonographers keep their eyes on the screen while their dominant hand moves the probe across the area of interest, while the other hand is used to optimise the digital image by adjusting the depth and gain. Findings can be compared with the contralateral side, which can be used as a control.

\section{Diagnostic Transthoracic Ultrasonography}

\section{The Normal Thorax}

The skin, muscles and facia planes are visible with a low-frequency probe as a series of echogenic layers (fig. 1) [1-3]. The ribs appear as convex structures on transverse (vertical) scanning, with posterior acoustic shadowing.
When the ribs are viewed longitudinally, the anterior cortex appears as a continuous echogenic line.

On low-frequency scanning, the visceral and parietal pleura appear as a single highly echogenic line no more than $2 \mathrm{~mm}$ in width, representing the pleura and pleuropulmonary interface [1-3]. On a longitudinal view, the pleural line will appear approximately $5-\mathrm{mm}$-deep to the rib cortex [8]. With high-resolution scanning, the visceral and parietal pleura can be seen as two distinct echogenic lines, with the latter seemingly thinner in appearance $[1,2]$. The two layers glide over each other during inspiration and expiration, which gives rise to the so-called 'lung sliding' sign on real-time US (online suppl. video 1; for all online suppl. material, see www. karger.com/doi/10.1159/000339997), best appreciated on longitudinal (vertical) scanning [1-4, 8]. Its presence has a high negative predictive value for the diagnosis of a pneumothorax $[4,8-10]$.

The diaphragm is best visualised through the liver or the spleen [1-4]. It appears as an echogenic 1- to 2-mm thick line which contracts with inspiration. In normal subjects, a 'curtain-sign' can often be seen at the costophrenic angle $[1,2]$; this refers to the variable obscuring of underlying structures by air in the lung. Abdominal viscera are visible on expiration, but during inspiration the normal aerated lung moves downwards in front of the probe, obscuring the sonographic window.

Normal lung parenchyma cannot be visualised on transthoracic US $[1-3,11]$. The large change in acoustic impedance at the pleura-lung interface, however, results in horizontal artefacts that are seen as a series of echogenic parallel lines equidistant from one another below the pleura (fig. 1) [1-3, 10]. These lines are known as reverberation artefacts or A-lines and diminish in intensity with increasing distance from the pleura. Short, vertical 'comet-tail' artefacts (fig. 1), presumably caused by fluidfilled subpleural interlobular septae, can be seen originating at the pleura-lung interface in normal individuals, and are best seen at the lung bases [1-3, 8]. B-lines are longer, vertical pathological artefacts (see below) that obliterate A-lines and move synchronously with lung sliding $[4,12]$.

\section{Extrathoracic Lymph Nodes, Chest Wall and \\ Diaphragmatic Pathology \\ Extrathoracic Lymph Nodes}

Cervical, supraclavicular and axillary lymph nodes are accessible by means of USwhich may aid in distinguishing reactive from malignant lymph nodes [4]. Inflammatory lymph nodes tend to have an echogenic fatty 


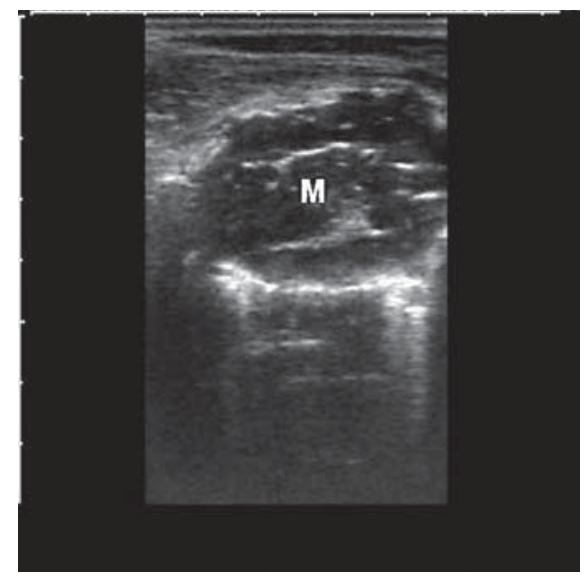

Fig. 2. An example of the US appearance of a chest wall mass (high frequency). In this particular case, US-assisted biopsy yielded a diagnosis of plasmacytoma. $\mathrm{M}=$ Mass.

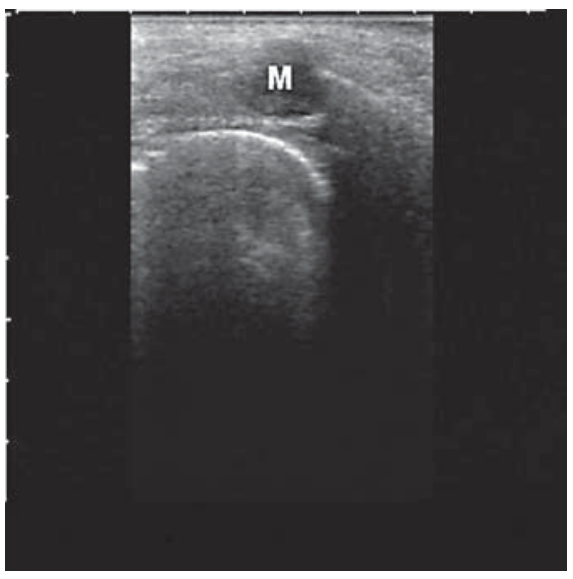

Fig. 3. The appearance of a rib metastasis (high frequency). This bony lesion was aspirated and found to be metastatic adenocarcinoma. $\mathrm{M}=$ Metastasis.

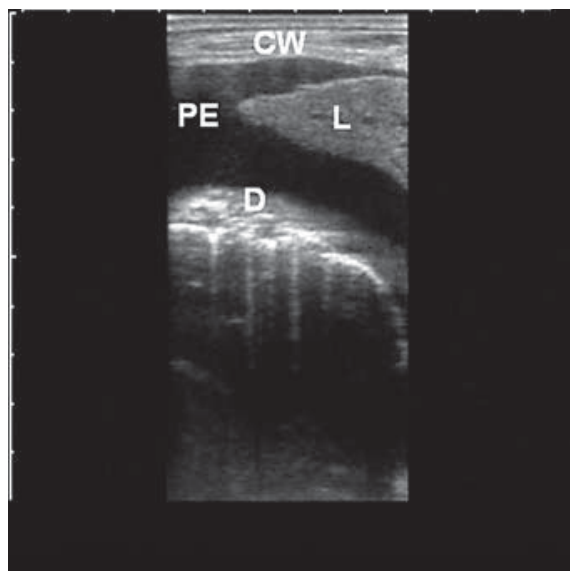

Fig. 4. A pleural effusion is presented as an anechoic space between the visceral and parietal pleura. Compressive atelectasis of the lung is seen on this high-frequency US. $\mathrm{CW}=$ Chest wall; $\mathrm{D}=$ diaphragm; $\mathrm{L}=$ lung; $\mathrm{PE}=$ pleural effusion. hilum and oval or triangular shape, whereas lymph nodes with malignant infiltration usually show loss of the fatty hilum leading to a hypoechoic appearance $[3,4,10]$. Malignant nodes also appear bulky, and extracapsular spread is suggested by irregular borders [13].

\section{Chest Wall Masses}

High-frequency US is ideally suited to visualise softtissue masses arising from the chest wall, e.g. lipomas, abscesses and many other (mostly benign) lesions. Masses generally have variable echogenicity and US findings (fig. 2) are too non-specific to differentiate between various aetiologies [1-3].

\section{Skeletal Pathology}

Sonography can detect bony metastases to the ribs (fig. 3), which appear as hypoechoic masses replacing the normal echogenicity of a rib and leading to the disruption of the cortical line [14]. Some evidence suggests that US may also be more sensitive than radiography in the detection of rib fractures [15], which appears as a breach or displacement of the rib cortex with or without a localized swelling or haematoma.

\section{Diaphragmatic Abnormalities}

The movement of the diaphragm should be unidirectional [1-3]. A degree of asymmetry in the movement of the hemidiaphragms is considered normal, but paradox- ical movement is indicative of a paralysed diaphragm [1$3,16]$. Forced inspiration ('sniff' test) is often utilized to accentuate this. US has been shown to be more accurate than fluoroscopy, and has the added advantage of being available at the point of care [8]. Long-term paralysis results in muscle atrophy [16].

\section{Pleural Pathology}

Pleural Effusions

Transthoracic US is ideal for the detection and quantification of pleural effusions, and is more sensitive than decubitus expiratory films in identifying minimal or loculated effusions [17]. A pleural effusion (fig. 4) gives rise to an anechoic, homogeneous space between parietal and visceral pleura. The space usually changes shape during respiration, although adhesions between the two pleural surfaces may result in the absence of lung motion above the effusion [1-3]. A tonguelike atelectatic lung is often seen inside a larger effusion.

The US appearance of a pleural effusion may vary depending on its nature, cause and chronicity. Four appearances are recognised based on the internal echogenicity: anechoic (fig. 4), complex non-septated, complex septated (fig. 5) and homogenously echogenic [1-3].

On US, transudates invariably give rise to anechoic and unseptated free-flowing effusions, whereas complex, septated or echogenic effusions are typically exudates [18, 19]. Malignant effusions are often anechoic, and pleu- 


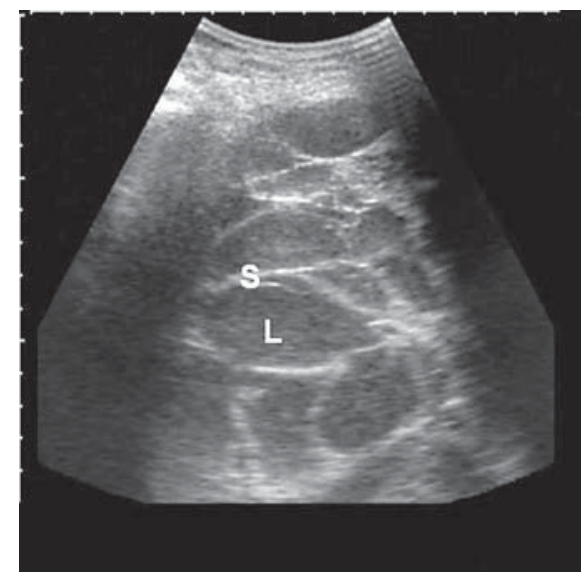

Fig. 5. A low-frequency US of a complex septated effusion with septae (S) and loculations (L).

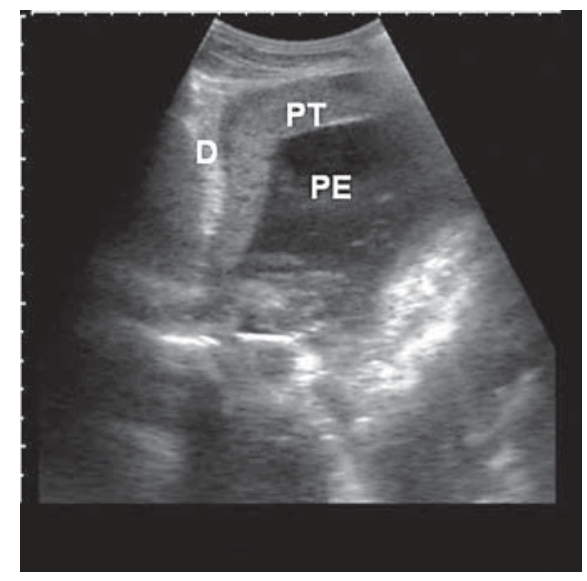

Fig. 6. A low-frequency US showing a pleural effusion associated with diffuse pleural thickening is suggestive of a malignant effusion. $\mathrm{D}=$ Diaphragm; PE = pleural effusion; $\mathrm{PT}=$ pleural thickening.

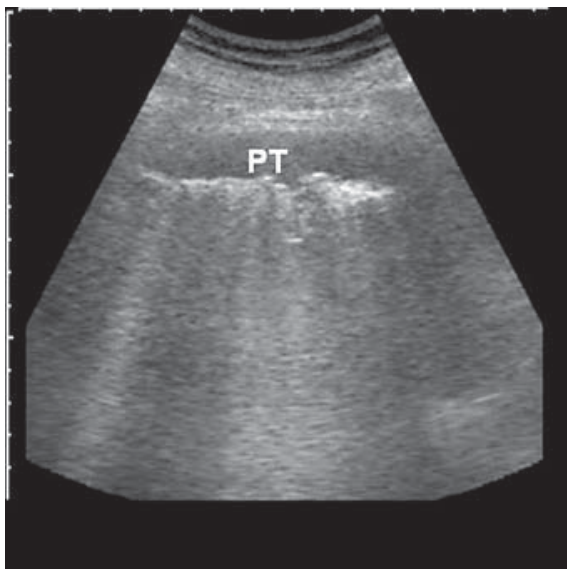

Fig. 7. Pleural thickening (PT). Note the hypoechoic appearance with distal enhancement (suggestive of chronicity). This patient was previously treated for tuberculous pleuritis. ral thickening $>10 \mathrm{~mm}$, pleural and diaphragmatic nodularity and diaphragmatic thickening $>7 \mathrm{~mm}$ (fig. 6) are highly suggestive of malignant disease [20]. In fact, Qureshi et al. [20] were able to identify 73\% of malignant effusions solely from their appearance on US. Inflammatory effusions are frequently associated with strands of echogenic material and septations which show more or less mobility with respiration $[1,2]$. The identification of septae has clinical implications. Several studies have shown that patients with septated effusions needed longer chest-tube drainage, longer hospital care, and are more likely to require fibrinolytic therapy or surgery compared to those with unseptated effusions [21, 22].

There is a reasonable correlation between the volume of an effusion estimated with planimetric measurements and its square dimensions [23-25]. Geometric calculations are often complicated by pleuropulmonary adhesions and the uneven distribution of fluid. Although not validated, a practical way to classify the volume of an effusion is simply to classify effusions as (1) minimal, if the echo-free space is confined to the costophrenic angle, (2) small, if the space is greater than the costophrenic angle but still within the range of the area covered with a 3.5$\mathrm{MHz}$ curvilinear probe, (3) moderate, if the space is greater than a 1-probe range but within a 2-probe range and (4) large, if the space is greater than a 2-probe range $[1-3,8]$.

Transthoracic US

\section{Pleural Thickening}

Pleural thickening (fig. 7) is often defined as a focal lesion arising from the visceral or parietal pleura that is greater than $3 \mathrm{~mm}$ in width with or without an irregular margin. Distinguishing pleural thickening from small effusions can be challenging, as both may appear hypoechoic on US. Pleural thickening does not display movement relative to the chest wall upon respiration and does not contain movable strands or echo densities [26]. Increased echogenicity with focal shadowing is sometimes observed and is indicative of calcification and chronicity. The fluid colour sign is the most sensitive and specific ultrasonographafic evidence to differentiate a small effusion from pleural thickening, and may be elicited with colour Doppler. Transmitted motion during respiratory or cardiac cycles causes a colour signal. In one study, it was shown to have a sensitivity of $89.2 \%$ and a specificity of $100 \%$ for the detection of minimal fluid collections [27].

\section{Pneumothorax and Hydropneumothorax}

The detection of a pneumothorax requires more experience than the detection of pleural fluid [1-3]. Nevertheless, a recent meta-analysis concluded that bedside ultrasonography performed by clinicians had a higher sensitivity and similar specificity, when compared to chest radiography in the diagnosis of a pneumothorax [28]. A pneumothorax can be reliably diagnosed by means of the absence of normal lung sliding and lung pulse, exagger- 
ated horizontal A-lines, the absence of B-lines and the presence of a lung point $[1,2,4,15,28]$. The 'lung pulse' refers to the subtle movements of the visceral pleura in relation to the parental pleura due to cardiac oscillations [4]. A 'lung point' is caused by the fact that the lung normally collapses to a dependent position posteriorly, and the air in the pleural space collects anteriorly in the supine patient. When a patient is scanned from anterior to lateral, a fleeting appearance of lung pattern may be observed (during inspiration) [4, 16, 28]. In an emergency setting, the absence of any movement in the pleural line (either lung sliding or pulse), coupled with the absence of B-lines allows prompt diagnosis of a pneumothorax, negating the need for searching for the lung point [4]. The sensitivity of US for the diagnosis of pneumothorax is limited in certain settings, including diaphragmatic paralysis, prior pleurodesis or pleural adhesions and emphysema $[1,2,16,28,29]$.

Ultrasonography is particularly useful in intensive care units and in other situations where radiographic equipment is not readily available or when CT scanning is impractical $[1,2]$. Furthermore, a pneumothorax following transbronchial biopsy can be reliably excluded with US (sensitivity $100 \%$ and specificity $83 \%$ ) [30]. Soldati et al. [31] showed that US was superior to chest radiographs in diagnosing pneumothoraces following blunt chest trauma. In their prospective study, they were able to show that a rapid US performed by an experienced operator had a sensitivity of $92 \%$ (spiral CT was used as the gold standard), whereas only $52 \%$ of pneumothoraces were visible on routine chest radiographs.

A hydropneumothorax may be identified with US by means of the visualisation of a dynamic air-fluid margin, with no sliding sign above the air-fluid level [32]. Furthermore, a 'curtain sign' may be present, which results from reverberation artefacts originating from the air within the pleura that obscures the underlying effusion during inspiration [32].

\section{Pulmonary Pathology}

Pneumonia and Lung Abscess

Provided aerated lung tissue is replaced by consolidated or solid lung tissue, practically any pathological process becomes detectable with US when pleural contact is present. 'Lung consolidation' is currently accepted as a non-specific term referring to a sub-pleural echopoor region or one with issue-like echotexture [4]. It can be caused by a variety of disease states, including pneumonia, pulmonary embolism, lung tumours, atelectasis and pulmonary contusion. Additional signs (e.g. the

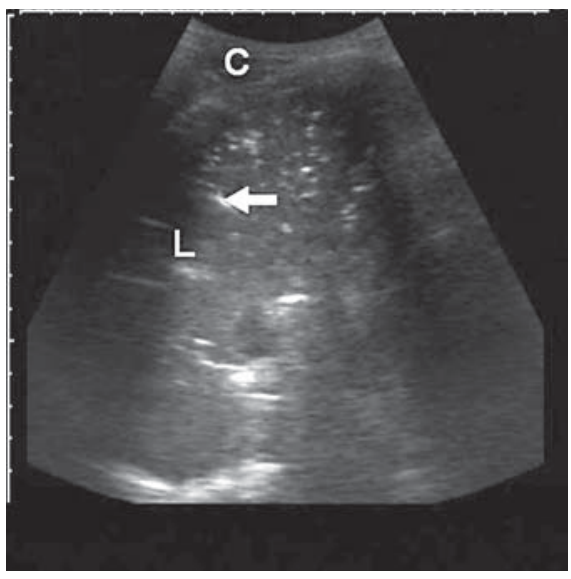

Fig. 8. A low-frequency US image of a densely consolidated lung $(\mathrm{L})$ abutting the chest wall $(\mathrm{C})$. Note the multiple air (hyperdense - arrow) and fluid (hypodense) bronchograms (best appreciated on real-time US).

quality of the deep margins, the presence of B-lines, air or fluid bronchograms and vascular pattern within the consolidation) may aid in distinguishing the various causes [4].

Pneumonic consolidation often appears less extensive on US than on chest radiograph. Early on, the parenchyma appears diffusely echogenic, similar to the ultrasonographic tissue-like texture of the liver. Both air and fluid bronchograms may be observed within the consolidated lung (fig. 8). Air bronchograms appear as echogenic branches and echogenic foci that fluctuate with respiration [1-4]. Multiple fleeting shadows are less commonly seen, and are sometimes referred to as 'dynamic' air bronchograms. Fluid bronchograms represent fluid-filled airways that are seen as anechoic tubular structures, and may be associated with bronchial obstruction or extensive pneumonic consolidation $[2,14]$. Furthermore, US is ideally suited to differentiate dense consolidation from pleural effusions in critically ill patients and to guide thoracocentesis in the same setting (online suppl. video 2).

A lung abscess that abuts the pleura appears as a hypoechoic lesion with a well-defined or irregular wall [13]. The centre of the abscess is most often anechoic, but internal echos may be seen. Abscesses with air fluid-levels are less homogeneous $[1,2]$.

\section{Lung Tumours}

Lung tumours are detectable provided they abut the chest wall $[1,2]$. Tumours often appear hypoechoic with posterior acoustic enhancement [3] (fig. 9). 

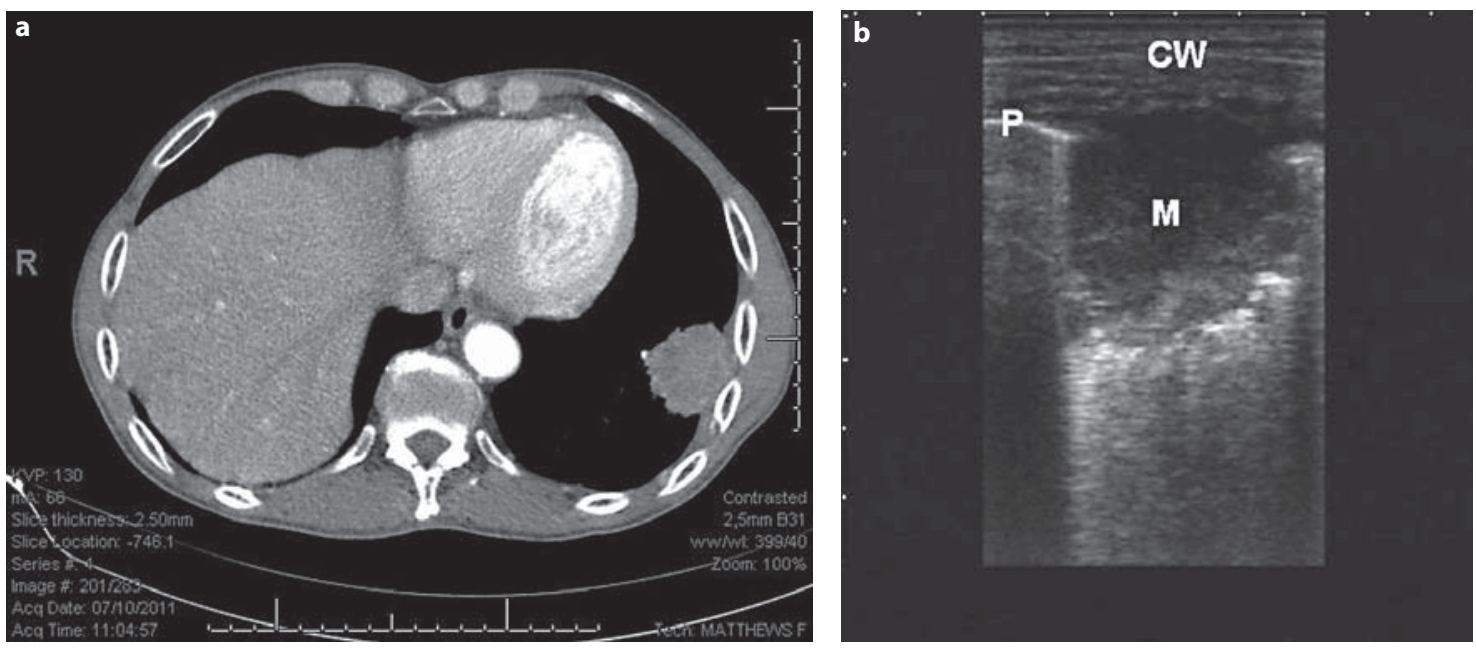

Fig. 9. A peripheral lung lesion with pleural contact. a CT scan image. b High-frequency US image. Note that the acoustic window is too narrow to demonstrate the whole circumference of the lesion, but it allows the determining of its full depth. Note the extension of the lung mass $(\mathrm{M})$ beyond the pleura $(\mathrm{P})$ into the chest wall $(\mathrm{CW})$. In this particular case, loss of movement with respiration was also demonstrated on real-time scanning, indicating chest wall invasion.

High-resolution US scanning is superior to routine chest $\mathrm{CT}$ in evaluating tumour invasion of the pleura and chest wall [33, 34]; this has important implications for lung tumour staging (T2 or T3 staging, respectively). Loss of movement with respiration on low- or high-frequency scanning also suggests extension beyond the parietal pleura $[1,2]$. Associated pulmonary collapse may cause fluid bronchograms $[2,35]$.

Colour Doppler US may distinguish malignant from benign pulmonary masses [36-38]. Approximately 65\% of peripheral malignant masses will demonstrate a colour Doppler signal (low-impedance flow) due to increased vascularity. A constant flow pattern correlates with malignancy, whereas a pulsatile or triphasic flow pattern is often observed in either benign or malignant mass lesions [36]. At least one study found that residual peripheral metastases demonstrated a reduced colour Doppler signal following chemotherapy [39].

Pulmonary Oedema and Other Interstitial Syndromes

B-lines (fig. 10) are long, laser-like vertical hyperechoic artefacts (considered pathological) that obliterate Alines, generally extend to the bottom of the screen and move synchronously with lung sliding [4]. The presence of multiple bilateral B-lines is indicative of an interstitial syndrome, which may include pulmonary oedema, interstitial pneumonia and diffuse parenchymal lung disease (pulmonary fibrosis) [4].
B-lines in the setting of patients with acute dyspnea has been reported to be a reliable sign to differentiate patients with pulmonary oedema from those with chronic obstructive airway disease [40]. 'Lung rockets' refer to the presence of several B-lines [16]. Lichtenstein et al. [41] found that B-lines were absent in $92 \%$ of patients with chronic obstructive airway disease, but present in $93 \%$ of patients with interstitial syndromes.

Acute respiratory distress syndrome tends to give rise to more patchy involvement (with spared areas) and a nonhomogeneous distribution of B-lines. Anterior subpleural consolidation may be seen, as well as reduced or even absent lung sliding and an irregular, thickened pleural line [4].

Multiple B-lines in a diffuse and nonhomogeneous distribution may be observed in patients with diffuse parenchymal lung disease (pulmonary fibrosis) [4]. Moreover, the distribution of B-lines correlates with CT evidence of fibrosis [4]. A fragmented pleural line and subpleural echo-poor areas are often present (in contrast to pulmonary oedema) [4].

\section{Pulmonary Embolism}

Pulmonary infarction may be visualised as a peripheral wedge-shaped hypoechoic region, frequently accompanied by a pleural effusion $[42,43]$. In fact, pulmonary embolism may be considered to be present when two or more characteristic triangular or rounded pleura-based 


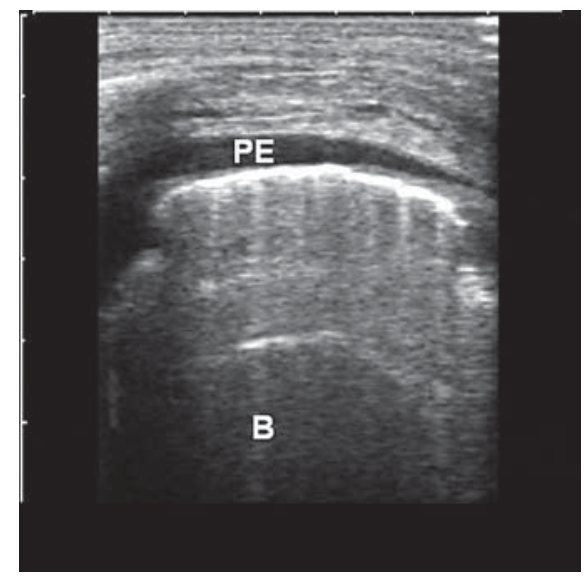

Fig. 10. A high-frequency US of a patient with pulmonary oedema showing widespread pronounced B-lines (a reliable sign of interstitial pulmonary oedema). $\mathrm{B}=\mathrm{B}$-lines; $\mathrm{PE}=$ pleural effusion.

lesions are present and probable if one typical lesion with a corresponding low-grade pleural effusion are present. Mathis et al. [43] prospectively applied the above-mentioned criteria in a large multicentre study $(n=352)$, and found transthoracic US to have a sensitivity of $74 \%$, a specificity of $95 \%$, a positive predictive value of $95 \%$ and a negative predictive value of $75 \%$ for pulmonary embolism.

The extent of pulmonary infarction may be underappreciated on US. In reality, the sonographic diagnosis of pulmonary emboli is currently only reserved for physicians with an interest in US, as CT angiography remains the investigation of choice. In certain circumstances (e.g. pregnancy), it may, however, be an invaluable diagnostic tool.

\section{Other Pathology}

Pulmonary cysts that abut the chest wall and pleuralbased cysts (e.g. hydatid cysts) can be visualised as large, round anechoic lesions (fig. 11) [2]. Pulmonary arteriovenous malformations appear as distinct hypoechoic lesions with posterior acoustic enhancement [44]. Lesions display high vascularity on Doppler, with low-impedance flow. Rounded atelectasis may be visualised as a pleural-based mass with associated pleural thickening and extrapleural fat. The invaginated pleura may result in an echogenic line running from the pleura into the mass [45].

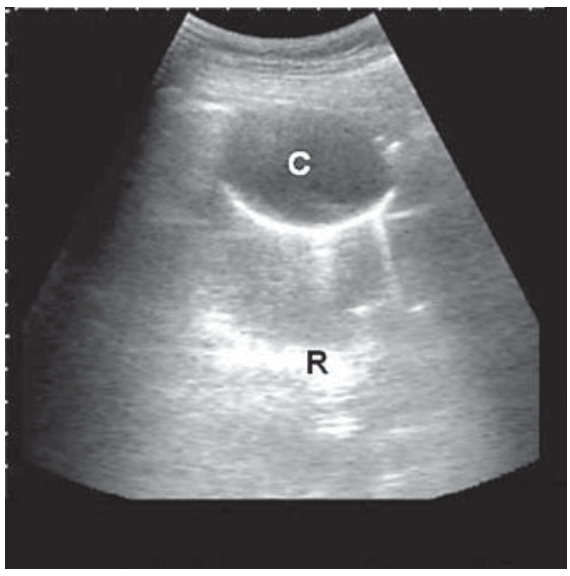

Fig. 11. This patient presented with a suspected hydatid cyst. An US-assisted fine-needle aspiration was safely performed. Note the reverberation artefact $(\mathrm{R})$ due to the high water content of the cyst (C).

\section{Ultrasound-Assisted Interventions}

\section{General Principles}

US is ideal for guiding numerous transthoracic procedures, as it potentially increases the success rate and minimises risk compared to blind procedures $[1,2,8]$.

Although specific reusable probes for real-time US guidance of needle biopsies are commercially available, most experienced clinicians utilize the 'freehand' technique. Following suitable patient positioning, the intended site of needle insertion is identified with US and marked, whilst the direction, depth of interest and safety range for the procedure are determined $[1,2]$. It is essential for the patient not to alter their position, in order to prevent a positional shift of the area of interest relative to the skin mark. Occasionally, it may even be necessary to ask the patient to hold his breath for the duration of the aspiration. The identification of a potential puncture site in a radiology department prior to transporting a patient elsewhere for thoracentesis or biopsy should be discouraged, particularly in the case of small effusions, as both the effusion and the skin mark may shift significantly with relatively minor changes in body position.

Transthoracic US is well validated as a screening method for post-procedural pneumothoraces, and should be performed in practically all patients after transthoracic procedures. In fact, US (specifically the loss of lung sliding) is potentially more sensitive than chest radiograph in the detection of pneumothoraces $[8,30,31]$. 


\section{Pleural Fluid Aspiration}

US is superior to chest radiograph for the determination of the optimal site for thoracentesis [2]. US guidance improves the success rate of pleural aspirations, which may be as high as 97\% [46]. US detects pleural fluid septations with greater sensitivity than CT scanning and also minimizes the risk of visceral puncture, which is particularly helpful when a safe procedure is mandatory, e.g. in patients with bleeding diathesis [46, 47]. Moreover, the risk of pneumothorax following aspirations is reduced, independent of the size of the effusion [47].

\section{Closed Intercostal Tube Drainage}

US is also ideal for identifying the optimal site for safe and effective intercostal drainage (ICD) and the current British Thoracic Society Pleural Disease guideline states that guidance by US is strongly recommended for all procedures involved in the drainage of pleural fluid [47, 48]. This is particularly relevant in patients with loculated parapneumonic effusions, where thickened parietal pleura, adhesions or loculations often complicate insertion [1-3]. Depending on operator experience, US may also guide further decisions regarding the need for intrapleural fibrinolytics, thoracoscopy or for surgical intervention in addition to tube drainage and antibiotics [49].

US-assisted drains are most often inserted by means of the 'freehand' technique (online suppl. video 3). The intended site should have a sufficient depth of pleural fluid of at least $10 \mathrm{~mm}$, no intervening lung and a minimal risk of visceral trauma [48]. Laceration of the intercostal neurovascular bundle may still occur, as these structures may have a torturous course that runs medially to the angle of the rib $[48,50]$.

As an alternative, effusions may also be aspirated and drained by means of an 18- or 16-gauge needle under real-time US guidance. This allows direct fluid aspiration followed by an insertion of a guide-wire, which is used to guide dilatation of a tract and deployment of a small-bore catheter (8- to 14-F). These tubes are better tolerated than large bore (20- to 24-F) intercostal drains [51], although common sense dictates that smaller bore drains are more likely to fail in the presence of pus with a high viscosity. However, some prospective studies have found that 8- to $12-\mathrm{F}$ pigtail catheters or 10 - to $14-\mathrm{F}$ catheters inserted with the Seldinger technique inserted under US guidance are at least as effective as larger catheters [52-54]. The positioning of the catheter tips with guidance is likely to be superior to a blind insertion, irrespective of drain size. Of note, most of the above-mentioned studies also em- ployed a strict rinsing schedule, which might be difficult to sustain in everyday clinical practice.

A survey carried out in the UK highlighted the dangers of blind pleural procedures: 67 of 101 National Health Service trusts reported at least one serious complication from ICD [55]. In all, 47 cases of serious lung or chest wall injuries with 8 deaths and 6 cases of ICD placement on the wrong side were described.

\section{Closed Pleural Biopsies}

Closed pleural biopsy needles were introduced in the 1950s, almost 4 decades after Hans Christian Jacobaeus established thoracoscopy [56]. Within a decade, various needles were described, of which the Abrams (guillotine) needle became the most widely used [56]. In the late 1980s, the use of unaided Tru-Cut needle biopsy was described as an alternative to Abrams needles in patients who present with large pleural effusions [57]. Closed pleural biopsy could conventionally be performed only in the presence of a moderate pleural effusion or pneumothorax. Unaided closed pleural biopsy is known to have a modest diagnostic yield of less than $60 \%$ for pleural malignancy [58]. Tuberculous granulomata are far more homogenously distributed over the pleura than is the case for metastatic deposits, and unaided closed biopsies are therefore more likely to be diagnostic if tuberculosis is the underlying disease [56]. The diagnostic yield of unaided closed pleural biopsy for pleural tuberculosis is generally in the order of $80 \%$, and may even be as high as $87 \%$ if at least six specimens are harvested [59-61].

Transthoracic US is an extremely helpful guide for biopsies of the pleura $[1-3,56]$. Focal pleural abnormalities can be identified with US, and biopsies can be aimed at areas of interest. Measuring the size of an associated effusion decreases the risk of visceral lacerations, which is particularly relevant in cases with small pleural effusions $[1,3]$. Recent studies have proposed that image-guidance may significantly increase the yield while decreasing the risk for complications [56, 62-65].

Chang et al. [64] found that the diagnostic yield of USguided Tru-Cut pleural biopsy to be as high as $87 \%$ for all pleural pathologies (77\% for malignancies). For malignant mesothelioma extending at least $20 \mathrm{~mm}$ in any accessible dimension on US, Diacon et al. [65] showed that this figure may be as high as $100 \%$.

In a randomized study, we found US-assisted Abrams needle biopsy specimens to be more likely to contain pleural tissue than specimens obtained with US-assisted Tru-Cut biopsies (91.0 vs. 78.7\%, $\mathrm{p}=0.015$ ) [62]. Abrams needle biopsies had a significantly superior yield for 
pleural tuberculosis compared to Tru-Cut needle biopsies ( 81.8 vs. $65.2 \%, p=0.022)$, but not compared to previously reported figures for blind Abrams needle biopsies, suggesting that US guidance offered little advantage beyond increased safety. Interestingly, and contrary to previous reports, the respective yield for both needle types for pleural malignancies was comparable and relatively high, with a diagnostic of $83.3 \%$ for US-assisted Abrams needle biopsy [62]. A possible explanation for the relatively high yield was the fact that low biopsy sites were utilized, as it is known that the lower thoracic parietal pleura (close to the diaphragm) is more likely to contain secondary spread from visceral pleural metastases [56]. Such an approach is achievable with US assistance, but not with digital percussion as a guide. Moreover, malignant disease tends to give rise to more focal pleural involvement which may be discernable with US.

\section{Transthoracic Fine-Needle Aspirations and Needle}

Biopsies of Chest Wall, Pleural and Pulmonary

\section{Tumours}

Chest wall and pleural-based tumours are detectable by US, as are pulmonary masses, provided that chest wall contact is present [1-3]. These tumours often appear hypoechoic with posterior acoustic enhancement and are suited for transthoracic US-assisted biopsy, as no aerated lung needs to be traversed during biopsy [1-5]. US-assisted transthoracic fine-needle aspiration (TTFNA) has the additional benefit of being able to be performed outside of theatre, even in a general ward. TTFNA is performed under local anaesthesia, ideally with a 22 -gauge injection-type or spinal needle $[1,3]$. Cutting-needle biopsy (CNB) follows the same principles as TTFNA, but the devices are more invasive and carry a higher risk of visceral or vascular trauma $[3,56]$.

Diacon et al. [65] found that US-assisted CNB has a sensitivity of approximately $85 \%$ for lung tumours abutting the chest wall and a $100 \%$ sensitivity for mesothelioma. They observed a pneumothorax rate of $4 \%$, and concluded that US-assisted CNB was safe in the hands of pulmonologists. In a more recent study, the sameinvestigators found that US-assisted TTFNA with rapid on-site evaluation by a cytopathologist of tumours abutting the chest wall had a yield of $82 \%$. CNB was shown to be diagnostic in $76 \%$, and the combined yield of CNB and TTFNA was $89 \%$ [66]. Both US-guided CNB and FNA had low complication rates, with pneumothoraces observed in 4 and $1.3 \%$, respectively $[65,66]$. Subanalyses of the data showed that US-guided TTFNA was superior to CNB in confirm- ing a diagnosis of epitheloid carcinomas of the lung (95 vs. $81 \%, p=0.006$ ), whereas $\mathrm{CNB}$ was superior in cases of non-carcinomatous tumours and benign lesions. Similar findings were reported by Schubert et al. [67]. Both investigators concluded that $\mathrm{CNB}$ may be reserved for cases where cytology is non-contributory and a diagnosis other than epithelial carcinoma of the lung cancer is suspected.

Contrast-enhanced sonography is sometimes employed as an additional biopsy guide for subpleural lung tumours [68]. The ability of this technique to visualise microcirculation enables the differentiation of viable from necrotic portions $[68,69]$. In a recent study, Cao et al. [69] confirmed that contrast-enhanced transthoracic sonography was able to optimize a potential biopsy site. They found a diagnostic yield with contrast-enhanced biopsy of $93.6 \%$, compared to $78.0 \%$ with non-contrast-enhanced biopsy $(\mathrm{p}<0.05)$ [69].

\section{Transthoracic Fine-Needle Aspirations and Needle} Biopsies of Mediastinal Tumours

In a landmark study from the late 1980s, Saito et al. [70] diagnosed 31 of 45 mediastinal masses by means of US-guided needle biopsies. In total, 13 of 15 patients with malignancies had diagnostic biopsies. Yang et al. [71] subsequently found Tru-Cut needles to have a diagnostic yield of $88.9 \%$ for mediastinal tumours. The same authors also pioneered the supraclavicular approach for USguided biopsies of superior mediastinal tumours [72]. Despite these encouraging findings, US-assisted mediastinal biopsy has not gained in popularity amongst clinicians, possibly because investigators generally utilize CNB only $[73,74]$ and are almost exclusively specialist interventional radiologists [75-77]. Studies that included more than 40 patients reported a $1-6 \%$ complication rate from $\mathrm{CNB}$, with pneumothoraces, haemothoraces and haemoptysis the most common serious complications [71-76].

In a recent study, we performed US-assisted TTFNA with rapid on-site evaluation on 45 consecutive patients, immediately followed by CNB in those cases where a provisional diagnosis of epithelial carcinoma or tuberculosis could not be established (provided a safety range could be assured) [78]. An accurate cytological diagnosis was made in 33 cases (73.3\%), and was more likely to be diagnostic in epithelial carcinoma and tuberculosis than all other pathology $(\mathrm{p}<0.001)$. CNB yielded a diagnosis in $88.2 \%$. Overall, $93.3 \%$ of patients were diagnosed by this sequential single-session approach. 
In an earlier study, we performed US-assisted biopsy on 25 consecutive patients with superior vena cava syndrome with an associated mass lesion that abutted the chest wall [79]. TTFNA had a diagnostic yield of $96 \%$, and that of US-assisted CNB was $87.5 \%$. The incidence of minor haemorrhage was $4 \%$ following TTFNA and $18.8 \%$ following CNB. Neither procedure resulted in major haemorrhage or pneumothoraces.

\section{Transthoracic Fine-Needle Aspirations and/or Needle}

Biopsies of Other Intrathoracic Pathology

The indications for US-assisted transthoracic biopsy are not limited to solid tumours or pleural disease. Yuan et al. [35] reported a microbiological yield of as high as 93\% with US-assisted biopsies of pulmonary consolidation of unknown aetiology. This procedure may be particularly useful in the immunocompromised patient, given the extensive differential diagnosis. The same authors were able to sonographically demonstrate abscess cavities in $94 \%$ of 35 patients with radiologically confirmed lung abscesses [80]. More than $90 \%$ of the aspirates from these abscesses yielded pathogens, whereas less than $10 \%$ of patients had positive blood cultures. Possible soiling of the pleural space is sometimes listed as a theoretical concern [8].

Proximal lung tumours are not discernable on US, but may cause varying degrees of pulmonary collapse (resorptive atelectasis) and postobstructive pneumonitis. 'Drowned lung' is a radiological term often used to describe these areas which are considered to represent accumulated secretions, and is typified on CT by enhanced pulmonary vasculature contrasted against surrounding pulmonary consolidation [81].

It is detectable on US, and typified by fluid bronchograms, provided the consolidation extends to the chest wall $[2,81]$. In a recent study, we found that US-assisted TTFNA of drowned lung yielded neoplastic cells in $74.2 \%$ of cases, and represented a viable alternative to bronchoscopy in some patients [79].

\section{Aspiration of Extrathoracic Lymph Nodes}

Although aspiration of extrathoracic lymph nodes is technically not a 'transthoracic' procedure, it deserves special mention. US-guided FNA of supraclavicular lymph nodes is practiced in many institutions, and has the advantage of providing a cytological diagnosis and pathological staging (pN3) all in one, minimally invasive procedure. A recent report emphasized the value of USguided FNA of supraclavicular lymph nodes: the procedure provided pathological diagnoses in $18.7 \%$ (95\% CI,
15.9-20.5) of all cases of lung cancer diagnosed over a 2 -year period $(\mathrm{n}=996)$ [82]. US has an added advantage in that it can be used to visualise cervical or supraclavicular lymph nodes in patients who present with superior vena cava obstruction, particularly when swelling and vascular congestion complicate routine physical palpation.

\section{Basic Competency in Ultrasonography}

Transthoracic US remains an operator-dependent modality during which image acquisition and interpretations occur simultaneously $[47,48,83]$. Recently, there have been renewed calls for formal instruction of basic transthoracic US skills to all respiratory physicians in training, for some form of certification in basic competency in ultrasonography and for the standardization of sonographic nomenclature [4, 48, 83-85]. The Critical Care Network of the American College of Chest Physicians in partnership with La Société de Réanimation de Langue Française relatively recently developed a consensus statement on competency in critical-care ultrasonography, and identified pleural and lung US as two of the core areas in the statement [83]. According to their statement, basic knowledge and skills are required in five principle areas: (1) basic US physics, (2) a knowledge of machine controls and transducer manipulation, (3) a knowledge of normal and abnormal anatomy and the pathophysiological consequences of observed abnormalities, (4) a knowledge of image interpretation, clinical application and the limitations of US and (5) a knowledge of when the examination is beyond the technical or interpretive capability of the clinician. The statement also described the basic technical (image acquisition) and cognitive (image interpretation) elements required for competency in both pleural and lung ultrasonography.

More recently, the British Thoracic Society endorsed the Royal College of Radiologist guidelines on the minimum standards to achieve a basic or level 1 competency in transthoracic US $[48,84]$. Level 1 competency includes (1) the recognition of normal anatomy of the pleural and diaphragm, (2) identification of the heart, liver and spleen, (3) recognition of a pleural effusion and estimating its depth, (4) differentiating a pleural effusion from pleural thickening, (5) identification of consolidated lung and (6) guided thoracocentesis and ICD placement. 
The minimum length of training and number of scans may vary amongst trainees, and there is currently little evidence in this regard [85]. The British Thoracic Society and Royal College of Radiologists suggest a minimum of 50 diagnostic scans and 5 interventions in order to achieve a basic (level 1) competency [84]. These procedures should be supervised and appropriately documented (a logbook should also be kept).

\section{Conclusions}

All training programs in respiratory medicine should include training in the basics (level 1) of transthoracic ultrasonography, given the growing body of evidence on its usefulness of as a diagnostic modality, and its ability to guide diagnostic and therapeutic interventions. The main indications of transthoracic US remain the investigation of chest wall abnormalities, pleural thickening and pleural tumours, and the qualitative and quantitative description of pleural effusions. US can visualise lung tumours, pulmonary consolidations and other parenchymal pulmonary processes provided they abut the pleura. It is the ideal tool to assist with thoracentesis and the drainage of effusions. US-assisted fine-needle aspiration and the CNB of lesions arising from the chest wall, pleura, peripheral lung and mediastinum are safe and have a high yield in the hands of chest physicians. It may also assist the aspiration and biopsy of diffuse pulmonary infiltrates, consolidations and lung abscesses, provided the chest wall is abutted. Other applications of transthoracic US include the diagnosis of a pneumothorax and pulmonary embolism.

\section{References}

1 Koegelenberg CFN, Bolliger CT, Diacon AH: 11 Tsai TH, Yang PC: Ultrasound in the diagnoPleural Ultrasound; in Light RW, Lee YC (eds): Textbook of Pleural Disease, ed 2. London, Hodder and Stoughton, 2008, pp 275283.

2 Koh DM, Burke S, Davies N, Padley SP: Transthoracic US of the chest: clinical uses and applications. Radiographics 2002;22:E1.

3 Koegelenberg CFN, Diacon AH, Bolliger CT: Transthoracic ultrasound of the chest wall, pleura, and the peripheral lung; in Bolliger CT, Herth FJF, Mayo PH, Miyazama T, Beamis JF (eds): Progress in Respiratory Research. Clinical Chest Ultrasound. Basel, Karger, 2009, vol 37, pp 22-33.

$\checkmark 4$ Volpicelli G, Elbarbary M, Blaivas M, et al: International evidence-based recommendations for point-of-care lung ultrasound. Intensive Care Med 2012;38:577-591.

5 Diacon AH, Theron J, Bolliger CT: Transthoracic ultrasound for the pulmonologist. Cur Opin Pulm Med 2005;11:307-312.

-6 Mayo PH, Doelken P: Pleural ultrasonography. Clin Chest Med 2006;27:215-217.

>7 Evans AL, Gleeson FV: Radiology in pleural disease: state of the art. Respirology 2004;9: 300-312.

8 Koenig SJ, Narasimhan M, Mayo PH: Thoracic ultrasonography for the pulmonary specialist. Chest 2011;140:1332-1341.

-9 Tsai TH, Jemg J-S, Yang P-C: Clinical applications of transthoracic ultrasound in chest medicine. J Med Ultrasound 2008;16:7-25.

10 Lichtenstein DA, Menu Y: A bedside ultrasound sign ruling out pneumothorax in the critically ill. Chest 1995;108:1345-1348. sis and management of pleural disease. Curr Opin Pulm Med 2003;9:282-290.

12 Lichtenstein DA, Mezière GA: Relevance of lung ultrasound in the diagnosis of acute respiratory failure: the BLUE protocol. Chest 2008;134:117-125.

13 Prosch H, Strasser G, Sonka C, Oschatz E, Mashaal S, Mohn-Staudner A, Mostbeck GH: Cervical ultrasound (US) and US-guided lymph node biopsy as a routine procedure for staging of lung cancer. Ultraschall Med 2007;28:598-603.

14 Mathis G: Thoraxsonography. I. Chest and pleura. Ultrasound Med Biol 1997;23:11311139.

15 Bitschnau R, Gehmacher O, Kopf A, Scheier M, Mathis G: Ultrasound in the diagnosis of rib and sternal fracture. Ultraschall Med 1997;18:158-161.

16 Gottesman E, McCool MD: Ultrasound evaluation of the paralyzed diaphragm. Am J Resp Crit Care Med 1997;155:1570-1574.

17 Kocijancic I, Kocijancic K, Cufer T: Imaging of pleural fluid in healthy individuals. Clin Radiol 2004;59:826-829.

18 Yang PC, Luh KT, Chang DB: Value of sonography in determining the nature of pleural effusion: analysis of 320 cases. AJR Am J Roentgenol 1992;159:29-33.

19 Hirsch JH, Rogers JV, Mack LA: Real-time sonography of pleural opacities. Am J Roentgenol 1981;136:297-301.

20 Qureshi NR, Rahman NM, Gleeson FV: Thoracic ultrasound in the diagnosis of malignant pleural effusion. Thorax 2009;64: 139-143.
21 Chen KY, Liaw YS, Wang HC, Luh KT, Yang PC: Sonographic septation: a useful prognostic indicator of acute thoracic empyema. J Ultrasound Med 2000;19:837-843.

22 Tu CY, Hsu WH, Hsia TC, Chen HJ, Tsai KD, Hung CW, Shih CM: Pleural effusions in febrile medical ICU patients: chest ultrasound study. Chest 2004;126:1274-1280.

23 Eibenberger KL, Dock WI, Ammann ME, Dorffner R, Hörmann MF, Grabenwöger F: Quantification of pleural effusions: sonography versus radiography. Radiology 1994;191: 681-684.

24 Lorenz J, Borner N, Nikolaus HP: Sonographic volumetry of pleural effusions. Ultraschall Med 1988;9:212-215.

25 Balik M, Plasil P, Waldauf P, Pazout J, Fric M, Otahal M, Pachl J: Ultrasound estimation of volume of pleural fluid in mechanically ventilated patients. Intensive Care Med 2006;32: 318-321.

26 Marks WM, Filly RA, Callen PW: Real-time evaluation of pleural lesions: new observations regarding the probability of obtaining free fluid. Radiology 1982;142:163-164.

27 Wu RG, Yang PC, Kuo SH, Luh KT: 'Fluid color' sign: a useful indicator for discrimination between pleural thickening and pleural effusion. J Ultrasound Med 1995;14:767-769.

28 Ding W, Shen Y, Yang J, He X, Zhang M: Diagnosis of pneumothorax by radiography and ultrasonography: a meta-analysis. Chest 2011;140:859-866.

29 Chan SS: The comet tail artefact in the diagnosis of pneumothorax. J Ultrasound Med 2002;21:1060. 
-30 Kreuter M, Eberhardt R, Wenz H, Schmitteckert H, Heußel CP, Herth FJF: The correct one is: diagnostic value of transthoracic ultrasound compared to chest radiography in the detection of a post-interventional pneumothorax. Ultraschall Med 2011;32:E20E23.

31 Soldati G, Testa A, Sher S, Pignataro G, La Sala M, Silveri NG: Occult traumatic pneumothorax: diagnostic accuracy of lung ultrasonography in the emergency department: Chest 2008;133:204-211.

- 32 Targhetta R, Bourgeois JM, Chavagneux R, Marty-Double C, Balmes P: Ultrasonographic approach to diagnosing hydropneumothorax. Chest 1992;101:931-934.

- 33 Sugama Y, Tamaki S, Kitamura S, Kira S: Ultrasonographic evaluation of pleural and chest wall invasion of lung cancer. Chest 1988;93:275-279.

-34 Suzuki N, Saitoh T, Kitamura S: Tumor invasion of the chest wall in lung cancer: diagnosis with US. Radiology 1993;187:39-42.

- 35 Yuan A, Chang DB, Yu CJ, Kuo SH, Luh KT, Yang PC: Color Doppler sonography of benign and malignant pulmonary masses. Am J Roentgenol 1994;163:545-549.

-36 Hsu WH, Ikezoe J, Chen CY: Color Doppler ultrasound signals of thoracic lesions: correlation with resected histological specimens. Am J Resp Crit Care Med 1996;153:19381951.

37 Hsu WH, Chiang CD, Chen CY: Color Doppler ultrasound pulsatile flow signals of thoracic lesions: comparison of lung cancers and benign lesions. Ultrasound Med Biol 1998; 24:1087-1095.

- 38 Liae YS, Yang PC, Yuan A: Ultrasonography and color Doppler imaging of metastatic pulmonary choriocarcinoma. Chest 1993; 104:1600-1601.

-39 Yang PC, Lee YC, Wu HD, Luh KT: Lung tumors associated with obstructive pneumonitis: US studies. Radiology 1990;174:717-720.

40 Lichtenstein D, Mézière G: A lung ultrasound sign allowing bedside distinction between pulmonary edema and COPD: the comet-tail artifact. Intensive Care Med 1998; 24:1331-1334.

-41 Lichtenstein D, Mézière G, Biderman P, Gepner A, Barré O: The comet-tail artifact: an ultrasound sign of alveolar-interstitial syndrome. Am J Respir Crit Care Med 1997;156 1640-1646.

42 Mathis G, Metzler J, Fubenegger D: Sonographic observation of pulmonary infarction and early infarctions by pulmonary embolism. Eur Heart J 1993;14:804-808.

43 Mathis G, Blank W, Reissig A, Lechleitner P, Reuss J, Schuler A, Beckh S: Thoracic ultrasound for diagnosing pulmonary embolism: a prospective multicenter study of $352 \mathrm{pa}$ tients. Chest 2005;128:1531-1538.
44 Wang HC, Kuo PH, Liaw YS: Diagnosis of pulmonary arteriovenous malformations by color Doppler ultrasound and amplitude ultrasound angiography. Thorax 1998;53:372376.

45 Marchbank MD, Wilson AG, Joseph AE: Ultrasound features of folded lung. Clin Radiol 1996;51:433-437.

46 Diacon AH, Brutsche MH, Solèr M: Accuracy of pleural puncture sites: a prospective comparison of clinical examination with ultrasound. Chest 2003;123:436-441.

47 Hooper C, Lee YC, Maskell N: Investigation of a unilateral pleural effusion in adults: British Thoracic Society pleural disease guideline 2010. Thorax 2010;65(suppl 2):ii4-iil7.

48 Havelock T, Teoh R, Laws D, Gleeson F: Pleural procedures and thoracic ultrasound: British Thoracic Society pleural disease guideline 2010. Thorax 2010;65(suppl 2):ii61-ii76.

49 Chen KY, Liaw YS, Wang HC: Sonographic septation: a useful prognostic indicator of acute thoracic empyema. J Ultrasound Med 2000;19:837-843.

50 Doelken P, Strange C: Chest ultrasound for 'dummies'. Chest 2003;123:332-333.

51 Tatersall DJ, Traill ZC, Gleeson FV: Chest drains: does size matter? Clin Radiol 2000; 55:415-421.

52 Shankar S, Gulati M, Kang M, Gupta S, Suri $S$ : Image-guided percutaneous drainage of thoracic empyema: can sonography predict the outcome? Eur Radiol 2000;10:495-499.

53 Ali I, Unruh H: Management of empyema thoracis. Ann Thorac Surg 1990;50:355-359.

54 Keeling AN, Leong S, Logan PM, Lee MJ: Empyema and effusion: outcome of imageguided small-bore catheter drainage. Cardiovasc Intervent Radiol 2008;31:135-141.

55 Harris A, O’Driscoll BR, Turkington PM: Survey of major complications of intercostal chest drain insertion in the UK. Postgrad Med J 2010;86:68-72.

56 Koegelenberg CF, Diacon AH: Pleural controversy: Closed needle pleural biopsy or thoracoscopy - which first? Respirology 2011;16:738-746.

57 McLeod DT, Ternouth I, Nkanza N: Comparison of the Tru-Cut biopsy needle with the Abrams punch for pleural biopsy. Thorax 1989;44:794-796.

58 Tomlinson JR: Invasive procedures in the diagnosis of pleural disease. Semin Respir Med 1987;9:30-60.

59 Valdés L, Alvarez D, San José E, Penela P, Valle JM, García-Pazos JM, Suárez J, Pose A: Tuberculous pleurisy: a study of 254 patients. Arch Intern Med 1998;158:2017-2021.

60 Diacon AH, Van de Wal BW, Wyser C, Smedema JP, Bezuidenhout J, Bolliger CT, Walzl G: Diagnostic tools in tuberculous pleurisy: a direct comparative study. Eur Respir J 2003;22:589-591.
61 Kirsch CM, Kroe DM, Azzi RL, Jensen WA, Kagawa FT, Wehner JH: The optimal number of pleural biopsy specimens for a diagnosis of tuberculous pleurisy. Chest 1997;112: 702-706.

62 Koegelenberg CF, Bolliger CT, Theron J, Walzl G, Wright CA, Louw M, Diacon AH: Direct comparison of the diagnostic yield of ultrasound-assisted Abrams and Tru-Cut needle biopsies for pleural tuberculosis. Thorax 2010;65:857-862.

63 Metintas M, Ak G, Dundar E, Yildirim H, Ozkan R, Kurt E, Erginel S, Alatas F, Metintas S: Medical thoracoscopy versus CT scan-guided Abrams pleural needle biopsy for diagnosis of patients with pleural effusions: a randomized, controlled trial. Chest 2010;137:1362-1368.

64 Chang BD, Yang PC, Luh KT, Kue SH, Yu CJ: Ultrasound-guided pleural biopsy with TruCut needle. Chest 1991;100:1328-1333.

65 Diacon AH, Schuurmans MM, Theron J, Schubert PT, Wright CA, Bolliger CT: Safety and yield of ultrasound assisted transthoracic biopsy performed by pulmonologists. Respiration 2004;71:519-522.

66 Diacon AH, Theron J, Schubert P, Brundyn $\mathrm{K}$, Louw $\mathrm{M}$, Wright CA, Bolliger CT: Ultrasound-assisted transthoracic biopsy: fineneedle aspiration or cutting-needle biopsy? Eur Respir J 2007;29:357-362.

67 Schubert P, Wright CA, Louw M, Brundyn K, Theron J, Bolliger CT, Diacon AH: Ultrasound-assisted transthoracic biopsy: cells or section? Diagn Cytopathol 2005;33:233-237.

68 Sartori S, Nielsen I, Trevisani L, Tombesi P, Ceccotti P, Abbasciano V: Contrast-enhanced sonography as guidance for transthoracic biopsy of a peripheral lung lesion with large necrotic areas. J Ultrasound Med 2004;23:133-136.

69 Cao BS, Wu JH, Li XL, Deng J, Liao GQ: Sonographically guided transthoracic biopsy of peripheral lung and mediastinal lesions: role of contrast-enhanced sonography. J Ultrasound Med 2011;30:1479-1490.

-70 Saito T, Kobayashi H, Sugama Y, Tamaki S, Kawai T, Kitamura S: Ultrasonically guided needle biopsy in the diagnosis of mediastinal masses. Am Rev Respir Dis 1988;138:679_ 684

-71 Yang PC, Chang DB, Yu CJ, Lee YC, Wu HD, Kuo SH, Luh KT: Ultrasound-guided core biopsy of thoracic tumors. Am Rev Respir Dis 1992;146:763-767.

72 Yang PC, Chang DB, Lee YC, Yu CJ, Kuo SH, Luh KT: Mediastinal malignancy: ultrasound guided biopsy through the supraclavicular approach. Thorax 1992;47:377-380.

73 Sawhney S, Jain R, Berry M: Tru-Cut biopsy of mediastinal masses guided by real-time sonography. Clin Radiol 1991;44:16-19. 
74 Samad SA, Sharifah NA, Zulfiqar MA, Maimunah A, Yahya A, Zainudin W: Ultrasound guided percutaneous biopsies of suspected mediastinal lesions. Med J Malaysia 1993;48: 421-426.

75 Andersson T, Lindgren PG, Elvin A: Ultrasound guided tumour biopsy in the anterior mediastinum. An alternative to thoracotomy and mediastinoscopy. Acta Radiol 1992; 33:423-426.

76 Ikezoe J, Morimoto S, Arisawa J, Takashima S, Kozuka T, Nakahara K: Percutaneous biopsy of thoracic lesions: value of sonography for needle guidance. Am J Roentgenol 1990; 154:1181-1185.

-77 Tikkakoski T, Lohela P, Leppänen M, ApajaSarkkinen M, Typpäo T, Mäkäräinen H: Ultrasound-guided aspiration biopsy of anterior mediastinal masses. J Clin Ultrasound 1991;19:209-214.
78 Koegelenberg CF, Bolliger CT, Irusen EM, von Groote-Bidlingmaier F, Mowlana A, Wright CA, Louw M, Schubert PT, Diacon $\mathrm{AH}$ : The diagnostic yield and safety of ultrasound-assisted biopsy of mediastinal masses. Respiration 2011;81:134-141.

79 Koegelenberg CF, Bolliger CT, Plekker DP, Wright CA, Brundyn K, Louw M, Schubert P, van den Heuvel MM, Diacon AH: Diagnostic yield and safety of ultrasound-assisted biopsies in superior vena cava syndrome. Eur Respir J 2009;33:1389-1395.

80 Yang PC, Luh KT, Lee YC: Lung abscesses: US examination and US-guided transthoracic aspiration. Radiology 1991;180:171175.

81 Koegelenberg CFN, Bolliger CT, Irusen EM, Wright CA, Louw M, Schubert PT, Diacon $\mathrm{AH}$ : The diagnostic yield and safety of ultrasound-assisted transthoracic fine needle aspiration of drowned lung. Respiration 2011; 81:26-31.
2 Hoosein MM, Barnes D, Khan AN, Peake MD, Bennett J, Purnell D, Free C, Entwisle JJ: The importance of ultrasound in staging and gaining a pathological diagnosis in patients with lung cancer - a two year single centre experience. Thorax 2011;66:414-417.

83 Mayo PH, Beaulieu Y, Doelken P: American College of Chest Physicians/La Société de Réanimation de Langue Française statement on competence in critical care ultrasonography. Chest 2009;135:1050-1060.

84 Faculty of Clinical Radiology: Ultrasound Training Recommendations for Medical and Surgical Specialities. London, Royal College of Radiologists, 2005.

-85 Kaplan A, Mayo PH: Echocardiography performed by the pulmonary/critical care medicine physician. Chest 2009;135:529-535. 